\title{
Signatures of the Youngest Starbursts: The Discovery of Ultradense H II Regions
}

\author{
Kelsey E. Johnson \\ JILA, Univ. Colorado, Boulder, CO 80309, USA
}

\section{Introduction}

Globular clusters are ubiquitous in the local universe, and their younger and bluer siblings, "super star clusters" (SSCs), have been observed in a large number of starburst galaxies. Recently a handful of ultra-young SSCs have been found still embedded in their birth material (e.g. Kobulnicky \& Johnson 1999 (here after KJ99); Turner et al. 2000; Beck et al. 2000; Tarchi et al. 2000). Because of their similarity (although on a vastly larger scale) to ultracompact $\mathrm{H}$ II regions in the Galaxy, KJ99 dub these embedded massive star clusters "ultradense HII regions" (UDH IIs). I will review the discovery of UDH IIs, their modeled properties, and their connection to the more familiar Galactic ultracompact $\mathrm{H}$ II regions.

\section{The Discovery of "Ultradense H II Regions"}

VLA radio continuum images have revealed compact radio sources in the starburst galaxy Henize 2-10. While the global radio continuum spectrum is indicative of non-thermal processes, the compact radio sources have positive spectral indices - suggesting an optically thick thermal bremsstrahlung origin. Moreover, these regions are not visible in optical images. After considering a variety of physical scenarios (such as supernovae or supernovae remnants, see KJ99), the most likely explanation is that these sources are dense HII regions surrounding massive star clusters. Therefore, we propose that the radio data preferentially reveal the youngest, densest, most highly obscured star-forming events.

\section{Modeled Properties}

Simple models of $\mathrm{HII}$ regions invoking free-free emission and self-absorption allow us to extract more information about the physical properties of these UDH IIs. As shown in Figure 1, sizes of $\approx$ a few parsecs and densities of $\approx$ $5000 \mathrm{~cm}^{-3}$ nicely reproduce the shape of the radio spectra. If we assume all of the $2 \mathrm{~cm}$ flux is thermal and a standard initial mass function, we can also estimate $\mathrm{N}_{\mathrm{Lyc}}$ and the masses of the UDH IIs. The resulting physical properties are truly remarkable (see KJ99 for more details); the estimated sizes (a few parsecs), masses (a few $\times 10^{5} \mathrm{M}_{\odot}$ ), excitations $\left(\mathrm{N}_{\mathrm{Lyc}} \sim 10^{51-52} \mathrm{erg}\right.$ ), and ages (less than a few $\times 10^{5}$ years) of the newly discovered UDH IIs imply that we may be witnessing the birth process of the familiar globular clusters. 


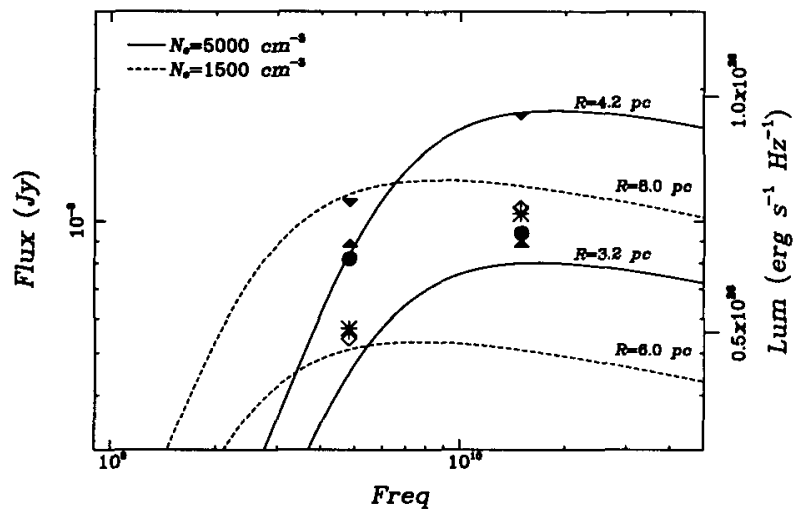

Figure 1. VLA $6 \mathrm{~cm}$ and $2 \mathrm{~cm}$ data for the five UDH IIs discovered by KJ99 in $\mathrm{He} 2-10$. The data are best modeled by sources with radii between 3 and $8 \mathrm{pc}$ and electron densities of $\approx 5000 \mathrm{~cm}^{-3}$.

\section{Connection to Ultracompact $\mathbf{H}$ II Regions}

There are a number of connections between UDHIIs and the UCHIIs. We already know that UCH IIs are often found in "complexes" (e.g. W49 or W51), so clustering in massive star formation is not a revelation. It seems likely that there is a continuum in the scale of massive star formation regions from single stars to UDH IIs. Also, based on dynamical arguments as well as number counts, we've estimated that UDH IIs spend $\approx 15 \%$ of their lifetimes enshrouded, which is nearly identical to the result found for UCH IIs (Wood \& Churchwell 1989). Based on these similarities, there is perhaps a parallel scheme for the evolution of single massive stars and clusters, and we can begin to observe UDH IIs with the same tools, techniques, and questions that have been applied to UCH IIs.

\begin{tabular}{|c|c|c|c|c|c|c|}
\hline Hot Cores & $\rightarrow$ & $\begin{array}{c}\text { UCH IIs } \\
\text { (Complexes) }\end{array}$ & $\rightarrow$ & $\begin{array}{c}\mathrm{OB} \\
\text { Associations }\end{array}$ & & \\
\hline $\begin{array}{c}\text { ???? } \\
\text { (not yet observed) }\end{array}$ & $\rightarrow$ & UDH IIs & $\rightarrow$ & $\begin{array}{l}\text { Super Star } \\
\text { Clusters }\end{array}$ & $\rightarrow$ & $\begin{array}{l}\text { Globular } \\
\text { Clusters }\end{array}$ \\
\hline
\end{tabular}

\section{References}

Beck, S.C., Turner, J.L., \& Kovo, O. 2000, AJ, 120, 244

Kobulnicky, H.A. \& Johnson, K.E. 1999, ApJ, 527, 154

Tarchi, A., Neininger, N., Greve, A., Klein, U. et al. 2000 A\&A, 358, 95

Turner, J.L., Beck, S.C., \& Ho, P.T.P 2000, ApJ, 532:L109

Wood, D.O. \& Churchwell, E. 1989, ApJS, 69, 831 